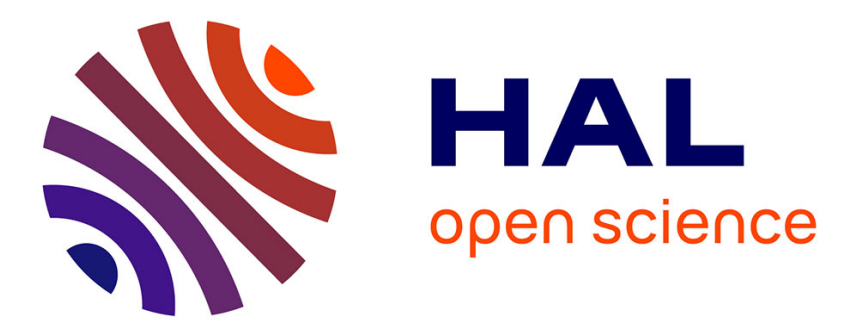

\title{
Experimental setup for transfer function measurement to assess RF heating of medical leads in MRI: Validation in the case of a single wire
}

Alexia Missoffe, Sarra Aissani

\section{- To cite this version:}

Alexia Missoffe, Sarra Aissani. Experimental setup for transfer function measurement to assess RF heating of medical leads in MRI: Validation in the case of a single wire. Magnetic Resonance in Medicine, 2017, 79 (3), pp.1766-1772. 10.1002/mrm.26773 . hal-03232480

\section{HAL Id: hal-03232480 \\ https://hal.univ-lorraine.fr/hal-03232480}

Submitted on 21 May 2021

HAL is a multi-disciplinary open access archive for the deposit and dissemination of scientific research documents, whether they are published or not. The documents may come from teaching and research institutions in France or abroad, or from public or private research centers.
L'archive ouverte pluridisciplinaire HAL, est destinée au dépôt et à la diffusion de documents scientifiques de niveau recherche, publiés ou non, émanant des établissements d'enseignement et de recherche français ou étrangers, des laboratoires publics ou privés.

\section{(c)(1)}

Distributed under a Creative Commons Attribution| 4.0 International License 


\section{Experimental Setup for Transfer Function Measurement to Assess RF Heating of Medical Leads in MRI: Validation in the Case of a Single Wire}

\begin{abstract}
Alexia Missoffe* and Sarra Aissani
Purpose: The measurement of the transfer function is a good tool to evaluate the radiofrequency heating of complex conductive wires, such as pacemaker leads. The aim is to describe precisely the design of a transfer function bench and compare the measurements to simulations.

Methods: The transfer function was measured by mean of an excitation probe and a receiving probe, both connected to a two-port vector network analyzer. The experimental results were compared with the simulated results, reproducing the excitation scheme. This procedure was applied to two different cables with different geometrical and insulation properties to test the robustness of the setup.

Results: It is possible to touch the cable electrode with the excitation probe without inducing an error in the measured transfer function, which solves the direct coupling problem. There is a good agreement between the measured and simulated transfer function for both tested cables.

Conclusions: A valid transfer function measurement bench is described. Magn Reson Med 79:1766-1772, 2018. (C) 2017 International Society for Magnetic Resonance in Medicine.
\end{abstract}

Key words: radiofrequency; transfer function; cables, MRI safety; heating; pacemaker leads

\section{INTRODUCTION}

Magnetic resonance imaging is an irreplaceable diagnostic tool that may become hazardous when patients are implanted with an active medical device-especially the radiofrequency $(\mathrm{RF})$ field, which presents a heating risk at the bare electrode of an implanted lead. The influence of various parameters on the lead heating has been reported in literature (1-9). For example, the resonance behavior as a function of the cable length has been studied with different geometrical parameters and material properties of the cable (1). The effect of the conductivity of the medium where the cable is embedded has also been studied (2). The influence of the position of a simple cable or a pacemaker lead in a phantom has also been reported $(3,4)$. The structure of different pacemaker/defibrillator leads has been studied (5), as well as the influence of the

IADI, U947, INSERM, Université de Lorraine, Nancy, France.

*Correspondence to: Alexia Missoffe, Ph.D., IADI, Université de LorraineINSERM, Bâtiment Recherche (anciennement EFS), Rez-de-Chaussé, CHRU de Nancy Brabois, Rue du Morvan, FR-54511 Vandoeuvre Cedex, France.

E-mail: alexia.missoffe@univ-lorraine.fr.

Received 1 February 2017; revised 10 May 2017; accepted 11 May 2017 DOI 10.1002/mrm.26773

Published online 6 June 2017 in Wiley Online Library (wileyonlinelibrary.com). termination conditions of pacemaker/defibrillator leads $(6,7)$. Among these studies, an important result is the influence of the phase distribution of the incident field on the lead heating $(8,9)$.

Indeed, a lot of the early studies analyzed the behavior of leads for a constant amplitude and constant phase incident electrical field (E-field) $(1,9)$, especially the behavior as a function of the length of the lead showing (in this case) a resonant effect. As mentioned previously, Yeung et al (8) showed the key importance of the phase distribution of the incident field on the possible heating at the electrode of a lead. Interesting experiments on cables have been made by Bottomley et al (9) to confirm this by folding cables along themselves with different folding lengths to change the incident field phase. These experiments show that a long cable that does not heat under a constant phase and constant amplitude incident field because it is above the resonant length can heat significantly once folded along a certain length. For example, in a human body, the incident field along a pacemaker lead has a distribution that is far from being constant (10) and is very different from the distribution in the American Society for Testing Material (ASTM) phantom (11) under the usual test conditions along the side of the phantom $(11,12)$. The incident field in a human body has a distribution so complex that it cannot be reproduced experimentally in a phantom. Therefore, the only way to estimate the heating of a lead in vivo is by simulating the lead in a human body model. Nevertheless, some leads such as pacemaker leads are too complex to simulate because of a very fine helicoidal structure (13). Park et al therefore introduced the transfer function concept (14). This concept comes directly from the formulation of the electromagnetic problem by the method of moments and it is implicit in the work by Yeung et al (8). The scattered field at the electrode is considered to be the linear superposition of the effect of the tangential incident field all along the cable given by the transfer function, which is a complex function of the position along the cable. This transfer function can be measured for complex cables that are too complicated to simulate. Knowing the incident field in a human model from a simulation, the heating can be estimated for a complex cable, thanks to the measured transfer function according to the following equation (14):

$$
\Delta T=\alpha\left|\int_{0}^{L} T F(z) E_{\text {inc }}(z) d z\right|^{2}
$$

where $\alpha$ is a calibration coefficient determined from an experiment in a phantom with a known incident field $E_{\text {inc }}(z)$ and $T F(z)$ is the complex transfer function at the position along the cable $z$. 
The problem arising is how to measure the transfer function. To reproduce what is physically happening, one would have to excite locally with an E-field along the cable and measure the scattered E-field at the electrode within a tissue-equivalent medium-filled tank. This can be done with a vector network analyzer (VNA) measuring the transmission coefficient S21 between the two probes. The signal that one wants to measure with the receiving probe is the signal from the cable excited by the excitation probe. In fact, the signal from the cable needs to be decoupled from the signal of the direct coupling between both probes. In other words, there are two coupling paths: one through the cable that reflects the transfer function signal, and the other one through the medium.

Feng et al. (15) proposed an alternative measurement based on the reciprocity principle conjugated with the Huygens principle. They developed the theory to show that in the case of a simple cable, if one excites the electrode where the heating has to be evaluated, the current distribution along the cable is actually the transfer function. They demonstrate first that the transfer function estimated this way by simulation does predict simulated scattered E-field for different incident fields on a simple cable. Second, they soldered a resistor at the end of more complex multiple wire cables and were able to predict the measured induced voltage with the measured transfer function. However, they do not describe their setup for measuring the transfer function based on the reciprocity approach. The measurements they have done on complex multiple wire cables validate the reciprocity approach for complex cables as well as their experimental setup. These cables were $48 \mathrm{~cm}$ long and were characterized by transfer functions defined all along these $48 \mathrm{~cm}$. They validate each of the functions by a comparison of nine measured induced voltages for nine different incident fields with the transfer function predictions. Nonetheless, several transfer functions could fit these measurement points. Therefore, it appears to be necessary to validate the experimental setup for the transfer function measurement on simple cables.

Nyenhuis et al (16) also presented a comparison between simulated and measured transfer function. Nevertheless, they reproduce their experimental scheme in the simulation with the aim of showing that the boundaries of the phantom of the setup influence the measured transfer function compared with an hypothetical infinite space. Their simulations can mimic an infinite space but are not free from the direct coupling problem between the probes. By using the Feng et al (15) approach, we simulated a transfer function by reproducing only the excitation scheme, so the simulations are free from the direct coupling problem. The added value of the simulations was to study how the position of the excitation probe influences the current along the cable and thus the measured transfer function.

Nyenhuis et al (17) very briefly described a transfer function setup designed with the direct approach in mind. It excites at a point along the cable by a so-called "toroidal coil," for which the dimensions are given but not the material nor the principle underlying the emission of the localized incident E-field field. The reception probe is described with no more information than as an "electrical field sensor." No information about the exact design nor the position of the probe is given. A measurement on a neurostimulator lead was made and a transmission line model was fitted to it. Nevertheless, no simulated theoretical transfer function of the lead was provided for comparison. In this article, we propose to validate a precisely described transfer-function measurement setup on a single wire by comparison with simulations that reproduce our excitation scheme. We verify that our current probe indeed measures the complex value of the current along the cable and that the coupling of the probes through the tissueequivalent medium is very small compared to the coupling through the cable. We did this on two different cables with different geometrical and material properties to check the robustness of our setup.

\section{METHODS}

\section{Measurement of the Transfer Function}

Figure 1 shows a global view of the setup as well as the excitation and receiving probes. The excitation probe was a monopole antenna made from a standard rg402 semirigid coaxial cable. Six millimeters of the core of the cable was left bare to constitute the monopole. For a better mechanical stability of the setup and to improve the coupling of the probes through the cable, the experimental excitation was done with the excitation monopole touching the electrode of the cable where the temperature measurements are usually done. As the transfer function depends on the termination condition of the cables on both ends, exciting the electrode this way might change the termination condition at the studied electrode end, and therefore the transfer function. Simulations have been done placing the excitation monopole transversally $0.5 \mathrm{~mm}$ away from the cable electrode and the transfer function fitted perfectly to the case where it touches to an amplitude scale factor and a constant phase shift close. These simulations justify the choice to make the excitation probe touch the electrode of the cable.

The receiving probe was also made from a rg402 semirigid coaxial cable on which a loop coil was soldered between the core and the shield. This loop coil was wound around a ferromagnetic torus (made of 4C65 ferrite which has an inner diameter of $8.2 \mathrm{~mm}$, an outer diameter of $14.6 \mathrm{~mm}$, and a height of $5.5 \mathrm{~mm}$ ). The cable to be studied was passed through the torus. This probe is based on the transformer principle, in which the cable is considered to be the primary winding and the loop coil the secondary winding. The loop coil was fixed to the torus with glue. The receiving probe was slid manually along the cable to measure the current every centimeter.

The measurements were done in an ASTM phantom filled to a height of $11 \mathrm{~cm}$ with saline solution, imitating the electrical properties of human tissues (11) (relative permittivity $\varepsilon_{\mathrm{r}}=80$, conductivity $\sigma=0.47 \mathrm{~S} / \mathrm{m}$ ). The cables were placed $6.5 \mathrm{~cm}$ above the bottom of the phantom, so $4.5 \mathrm{~cm}$ from the surface of the saline solution. They were placed in the middle of the width of the phantom, and the excited electrode was placed $17 \mathrm{~cm}$ from the bottom of the phantom. 

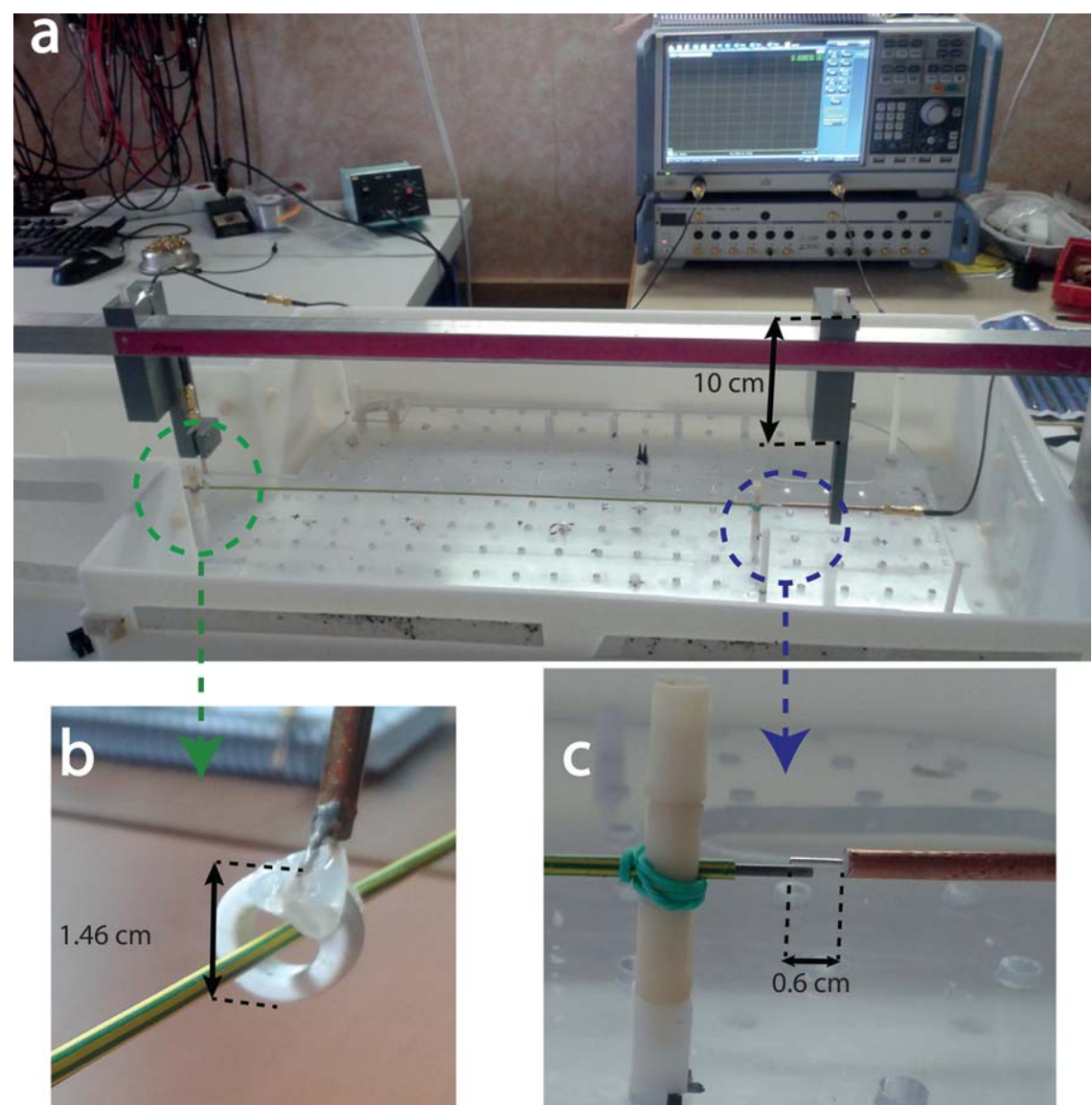

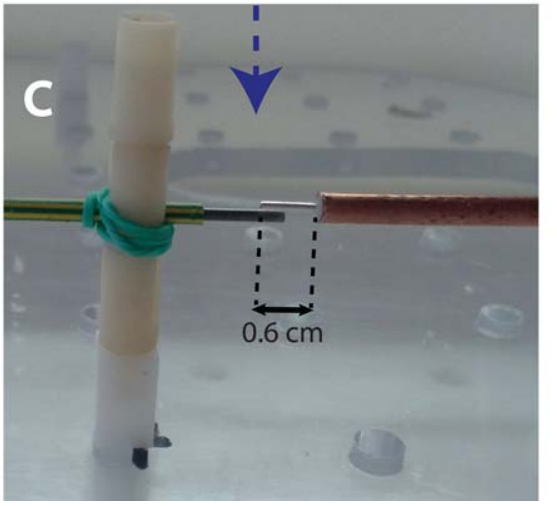

FIG. 1. Transfer function measurement setup. a: Global view with the VNA. b: The receiving probe that measures the current along the cable. c: The excitation probe with a cable.
A two-port VNA (ZNB4 Rohde \& Schwarz, Munich, Germany) was used for measurement. Calibration was done using the ZV-Z135 calibration kit 3.5 MM (F) on both ports according to the Through, Open, Short, and Match procedure. The calibration plane was brought to the end of two SMA male cables where the excitation and receiving probes were connected to the VNA port 1 and port 2, respectively. The complex S-parameter S21 was measured with the VNA for different positioning of the receiving probe along the cable with a step of $1 \mathrm{~cm}$. The cables were fixed on both ends, therefore keeping us from measuring the transfer function up to the ends (Fig. 1c). The output power of the VNA was set to $10 \mathrm{dBm}$.

When the two probes are close, the direct coupling between both probes was evaluated. This direct coupling was measured with a cable fixed in the middle, enabling us to bring the receiving probe to the end of the insulation, which would be the closest measurement point to the excitation electrode. At the position where the reception probe is at the end of the cable, there was a factor of 10 on the amplitude of S21 between the case where the cable was present $(-20 \mathrm{~dB})$ or not $(-40 \mathrm{~dB})$ and a factor of 180 at the previous position $1 \mathrm{~cm}$ away from the closest position. Therefore, the direct coupling is considered negligible, and the measurements close to the excitation electrode are considered reliable. There is no need to make a correction because of the direct coupling between probes.

\section{Cables}

The first cable was a 46-cm standard rg402 semirigid coaxial cable removed from its metallic shield on its length. Forty-six centimeters is about the wavelength at $64 \mathrm{MHz}$ in the saline solution. The cable insulator was stripped at both ends on a distance of $5 \mathrm{~mm}$ to constitute the electrodes where the scattered E-field is to be evaluated.

The electric properties at $64 \mathrm{MHz}$ of this standard cable are known, especially the relative permittivity $\varepsilon_{\mathrm{r}}$ of the Teflon insulation material, which has a value of 2.1 as mentioned in the data sheet. The measurement for this cable was made four times.

The second cable studied was a 53-cm-long and 1.5mm-diameter steel cable insulated with a $0.51 \pm 0.1 \mathrm{~mm}$ thick heat-shrinkable tube made of polyolefin. One centimeter of the cable was left bare at both ends to constitute the electrodes. Information found on a website (18) mentioned an hypothetical relative permittivity of 2.35 for polyolefins, although the frequency-range validity of their measurement was not given. Polyolefin is a generic term and the permittivity would need to be measured using a dedicated setup. Such a measurement was beyond the scope of this paper. To compensate for this missing information, we simulated the transfer function on the expected range of the insulation permittivity. This also provides information about the sensitivity of the transfer function to this property. 
The first cable was a semirigid cable that was difficult to maintain perfectly straight. This is an issue in the mechanical setup as the measurement probe was slid along a straight bar. The setup was, as much as possible, made so that the torus does not exert any constraint on the cable. Constraints on the cable might change the gain measurement artificially by slightly modifying the contact between the excitation probe and the cable electrode although the cable was fixed at the end. The second cable was perfectly straight, which made the measurement more reliable with potentially less uncertainty. The final purpose of this setup was to measure the transfer functions on pacemaker leads. Pacemaker leads are completely flexible, and if the end of the cable is fixed, a constraint along the length of the cable by the torus will have no influence on the contact between the excitation probe and the lead electrode.

\section{Simulations}

The simulations were made using CST MWS software (CST, Darmstadt, Germany). The excitation setup was reproduced with the exact position of the cable in the ASTM phantom and with the excitation probe touching the cable's electrode.

Nyenhuis et al (16) simulated their transfer function setup that is based on the direct approach and showed that the phantom boundaries have a very important influence on the measured transfer function. The transfer function calculated by the method of moments $(8,14)$ is the transfer function in a volume of a tissue-imitating medium considered to be infinite. The reciprocity approach allows to simulate a theoretical transfer function for any boundary conditions. Simulating the transfer function in an ASTM phantom that imitates the shape of a torso is more realistic than considering the surrounding tissue-imitating medium to be infinite.

It is important to mesh quite finely the electrode along the cable to simulate correctly the termination conditions. The mesh step along the cable was $2 \mathrm{~mm}$, and $0.5 \mathrm{~mm}$ along the electrode. The complex current distribution along the cable at $64 \mathrm{MHz}$ was the result the measurements were compared to. For the second cable, the simulations were made for different relative permittivities of the insulation material 2.2, 2.3, 2.35 and 2.4.

\section{Validation}

For the first cable, the mean and standard deviation of the amplitude and phase of the transfer function were calculated. The four measured transfer functions were each normalized according to Equation [2] and the mean amplitude was calculated on the normalized transfer functions. The scaling of the phase shift was made by fixing the phase at the electrode at zero which is the usual convention (14).

$$
T F_{N}(z)=\frac{T F_{\text {measured }}(z)}{\left|\int_{\mathrm{z}_{1}}^{z_{2}} T F_{\text {measured }}(z) d z\right|}
$$

Normalizations for the first and second cables were made on the length of the cable where the transfer function was actually measured.
To use the transfer function for heating evaluation, a calibration factor is determined experimentally by a heating experiment for a known incident field using Equation [1]. If we want to put the measured and simulated transfer function to scale, we can calculate the ratio of the calibration factors for both transfer function supposing the same calibration experiment was used. The ratio of the calibration factors is given by the following equation as the measured temperature increase is the same:

$$
\frac{\alpha_{C S T}}{\alpha_{\exp }}=\frac{\left|\int_{0}^{L} T F_{\exp }(z) E_{\text {inc1 }}(z) d z\right|}{\left|\int_{0}^{L} T F_{C S T}(z) E_{\text {inc1 }}(z) d z\right|}
$$

If we consider the normalized simulated and measured transfer functions and a constant amplitude and phase incident field, we find that the calibration factors are equal. Therefore, normalizing the transfer functions for their comparison is equivalent to considering that the calibration factors have been calculated for a constant amplitude and phase electrical incident field. Therefore, the normalized functions are to scale.

To assess the reliability of the measured transfer function compared with the simulation transfer function for heating prediction, the estimated scattered fields for three different incident fields were calculated. This was done only for the first cable.

The transfer function along the whole length of the cable is necessary, and an estimation of the missing measurement points had to be made. The simulated transfer function showed a linear behavior at the end of the cable opposite to the excitation probe. Therefore, the missing $2.2 \mathrm{~cm}$ at this end of the measured transfer function were linearly extrapolated.

In contrast, at the excited end of the cable, the simulated transfer function showed discontinuities in the derivative at $1.3 \mathrm{~cm}$ from the beginning of the insulation, which can be explained by the fact that the excitation probe excites the cable through the direct contact and through a radiated field. This is consistent with the evaluation of the radiated field from the emission probe being received directly by the reception probe without the presence of the cable under test. Therefore, at the excited end, the simulated transfer function was linearly extrapolated on the last $1.3 \mathrm{~cm}$. The measured transfer function was also linearly extrapolated on the missing $2 \mathrm{~cm}$. Another normalization was performed on the new transfer functions defined along the whole length.

Three different incident E-fields were extracted from a $1.5 \mathrm{~T}$ birdcage simulation with an ASTM phantom (11) placed such that the center of the torso was at position $\mathrm{x}=0 \mathrm{~mm}, \mathrm{z}=0 \mathrm{~mm}$, and $\mathrm{y}=-55 \mathrm{~mm}$. The position of the cable would be in the middle of the phantom on the $\mathrm{y}$ - and z-axis and at positions -165 , -90 , and $-15 \mathrm{~mm}$ on the $\mathrm{x}$-axis. Figure 2 illustrates the simulated setup.

\section{RESULTS}

Figure 3 shows the amplitude and phase distribution of the simulated and measured transfer functions for the first cable. The difference on the amplitude distribution 


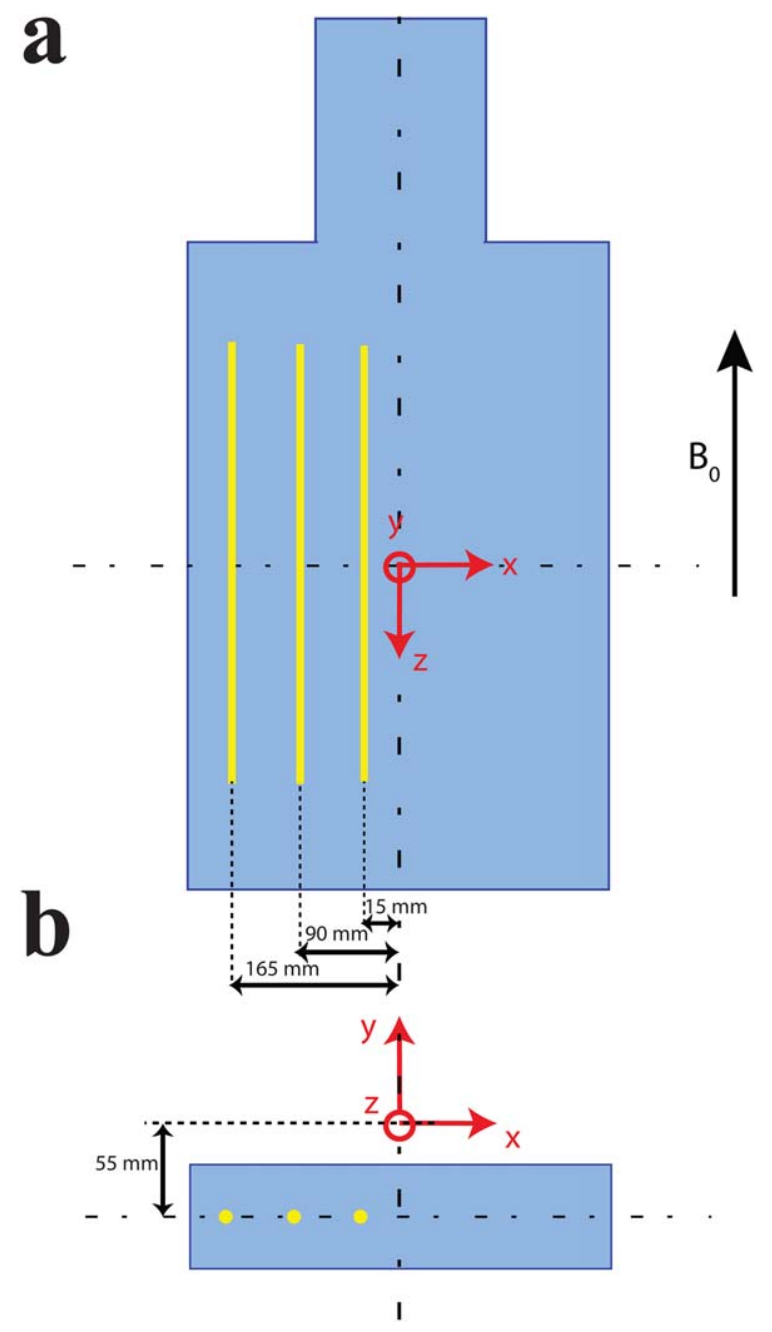

FIG. 2. Description of the simulation setup for extraction of the possible incident fields in an ASTM phantom. a: Top view. b: Front view.

is $2.4 \%$ and $7.2 \%$ on the phase distribution. The maximum standard deviation for the amplitude is 0.0275 , which is approximately $1 \%$ of the order of magnitude of the normalized amplitude, and $3.9 \%$ of the maximum variation of amplitude between two points. The maximum standard deviation on the phase is $1^{\circ}$ which is $1.7 \%$ of the total variation of the phase along the cable.

Table 1 lists the predicted scattered E-field for both transfer functions. The predicted scattered E-fields differ by less than $1 \%$ for the three incident fields. Although the measured and simulated transfer functions differ slightly, it is shown here that the result on the predicted scattered E-field at the electrode, and therefore the temperature elevation, is the same for both functions for three quite different incident field distributions.

Figure 4 shows the measured and simulated amplitude and phase of the transfer function for four different permittivities of the insulation. The relative difference for the expected permittivity of 2.35 is $4.8 \%$ on the amplitude distribution and $5.2 \%$ on the phase distribution. The measurements coincide best with the simulation for a permittivity of 2.2 , with a relative difference of $3 \%$ on the amplitude distribution and $2.6 \%$ on the phase distribution.
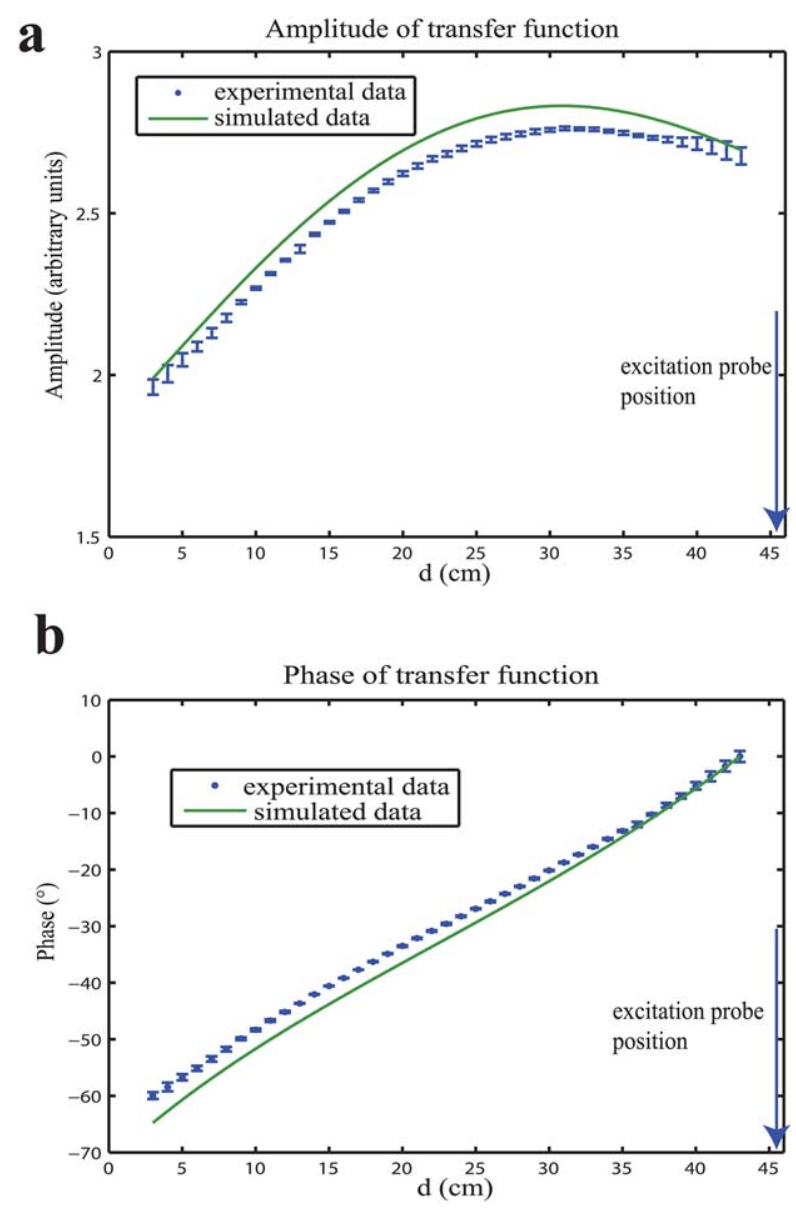

FIG. 3. Comparison between the experimental and simulated transfer functions for the $46-\mathrm{cm}$ rg402 coaxial cable with the shield removed and uncapped at both ends. a: Transfer functions amplitude. b: Transfer functions phase.

\section{DISCUSSION}

We presented a simple setup to measure the transfer function of a cable, and performed measurements on two different single wires with different geometrical and material properties. The precise designs of the probes were described such that the setup can be reproduced.

Simulation of the excitation probe allowed us to demonstrate that the excitation probe can be in contact with the cable electrode. This solves the problem of the direct coupling between the excitation and receiving probes which might be an issue in other designs.

The simulated and measured transfer functions for the first cable were in good agreement, so we can conclude that the simple current probe is reliable and that the

Table 1

Predicted Scattered E-Field at the Electrode by the Simulated and Measured Transfer Functions.

\begin{tabular}{cccc}
\hline & $\begin{array}{c}\text { E incident 1 } \\
\mathrm{x}=-165 \mathrm{~mm}\end{array}$ & $\begin{array}{c}\mathrm{E} \text { incident 2 } \\
\mathrm{x}=-90 \mathrm{~mm}\end{array}$ & $\begin{array}{c}\mathrm{E} \text { incident 3 } \\
\mathrm{x}=-15 \mathrm{~mm}\end{array}$ \\
\hline $\begin{array}{c}\text { E experimental } \\
(\mathrm{V} / \mathrm{m})\end{array}$ & 1.2091 & 0.7046 & 0.1441 \\
$\begin{array}{c}\mathrm{E} \mathrm{simulated} \\
(\mathrm{V} / \mathrm{m})\end{array}$ & 1.2121 & 0.7066 & 0.1444 \\
\hline
\end{tabular}



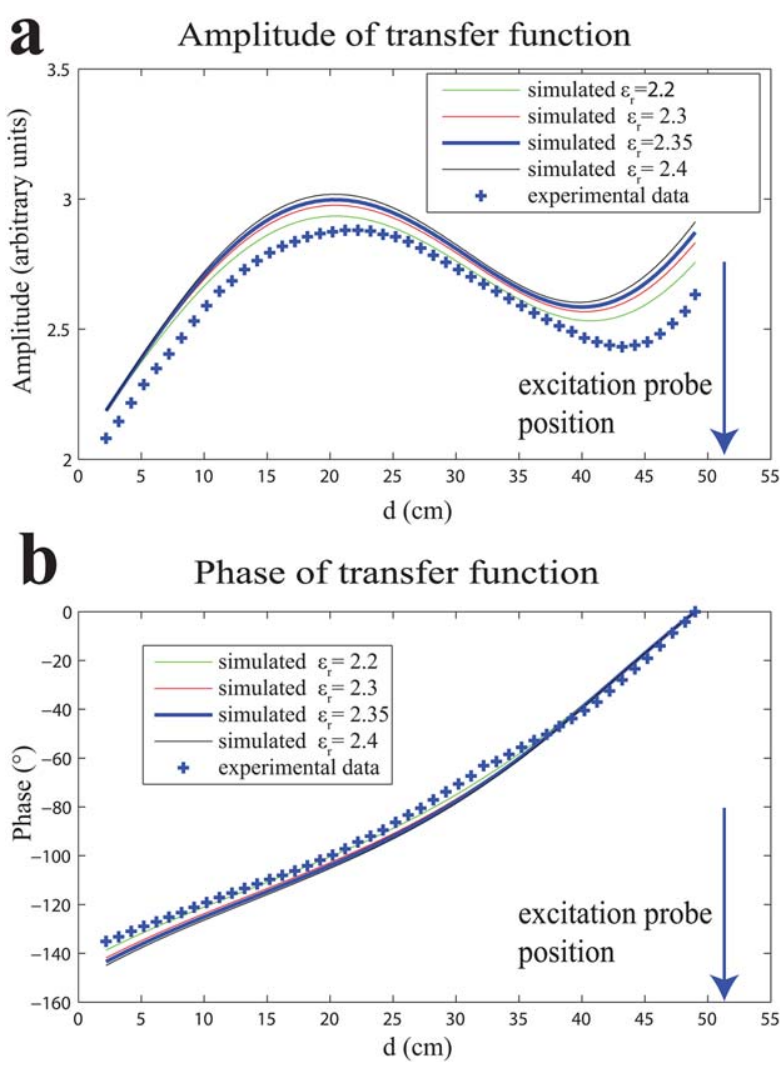

FIG. 4. Comparison between simulated transfer functions with different permittivities of the insulation and the measured transfer function for the 53-cm steel cable uncapped at both ends. a: Transfer functions amplitude. b: Transfer functions phase.

setup is indeed free from the direct coupling problem. It was shown that the slight difference would not affect the predicted heating for three different incident fields.

The measurement for the second cable also coincides very well with the simulations covering the range of the expected insulation permittivity, therefore confirming the robustness of the setup.

\section{Reciprocity Approach}

The design of the probes was based on the reciprocity approach. Therefore, we aimed at exciting the electrode and measuring the current along the cable. Nevertheless, the resulting network is linear therefore reciprocal. Indeed, we verified that the S12 parameter along the cable is exactly equal to the S21. We could therefore consider our current probe as a transmit antenna generating a localized E-field tangential to the cable and our monopole as an E-field sensor.

\section{Extrapolation at the Ends}

The fact that measurement points are missing at the end of the cable is due to the mechanical constraints of the actual setup. This is indeed an issue for measurement on pacemaker leads for which the behavior is highly unpredictable. Nevertheless, the mechanical setup could be improved in the future by redesigning it to allow greater freedom in the positioning of cable fixtures to allow measurements along the entire cable length.

\section{Matching of the Probes}

The calibration plane was at the end of the SMA male cables. As the probes were not matched, there were reflections at this plane. Nevertheless, this mismatch is not important as long as it is constant for both probes as the current probe is slid along the cable. Indeed, the transfer function measurement is a relative measurement at each position.

The measurement of the S11 of the monopole with the setup in place for the $53-\mathrm{cm}$ cable indicated that less than $3 \%$ of the input power was reflected, and that the variation of this reflected power during the measurement is negligible.

The potential variation of the S22 of the current probe could also influence its sensitivity and therefore make the measurement erroneous. We measured the S22 of the current probe for every position along the 53-cm cable. The results showed that approximately $30 \%$ of the power was reflected. The variation of the S22 reveals a maximum variation of the reflected power of $10 \%$ and therefore a maximum variation of the transmitted power to the cable and surroundings of $4 \%$. We consider that this slight variation does not affect the reliability of our measurements.

Matching the receiving probe at $64 \mathrm{MHz}$ would improve the signal-to-noise ratio, but is not mandatory regarding the high precision VNA used (dynamic range of $140 \mathrm{~dB}$ ).

\section{Validation of the Setup for Multiple Cables}

As mentioned, the transfer function method is meant to be used to evaluate the heating of leads that cannot be simulated such as pacemaker leads. Often, commercial medical device leads such as pacemaker ones are made of multiple coaxial helicoidal wires for which the theory of the reciprocity principle has not been demonstrated. Feng et al (15) demonstrated experimentally the validity of this approach for multiple cables stacked or a coaxial cable. As mentioned in the introduction, nine measurement points validate the transfer function on a length of $48 \mathrm{~cm}$, which corresponds to an undetermined problem. Nevertheless, our measurement showed that our setup, based on the reciprocity approach, can also be considered as a direct approach setup as it is completely reciprocal. Therefore, our setup that was designed with the reciprocity approach in mind is also valid for measurement on a complex pacemaker lead, although the reciprocity approach as presented by Feng et al (15) was not proved theoretically for complex cables.

\section{CONCLUSIONS}

It was shown that a simple setup can measure the transfer function of a cable. The proposed excitation setup is particularly relevant, as it enables to make the direct coupling between the probes negligible compared to the coupling through the cable. A very cheap (25€ for the rg402 cable and $1 €$ for the ferroxicube torus) and reproducible current probe design was proposed. This setup could be used for 
pacemaker leads in the future. Further work has to be carried out to validate this setup at $128 \mathrm{MHz}$.

\section{ACKNOWLEDGMENTS}

The authors thank Mr. Marande from CRM2 laboratory (Nancy, France) for his work on the mechanical setup, and Luc Chassagne from the LISV laboratory (Versailles, France) for his contribution to the excitation probe design. They wish to thank Thierry Ditchi from the LPEM laboratory (Paris, France) for the useful discussion about the insulation relative permittivity measurement, as well as Pierre André Vuissoz, Jacques Felblinger, and Julie Kabil from the IADI laboratory (Nancy, France) for the useful discussions. Finally, the authors thank the aunt of the first author, Colette Thomson, for correcting the English and Jacques Felblinger and Cédric Pasquier for the funding through Region Lorraine and FEDER.

\section{REFERENCES}

1. Yeung CJ, Susil RC, Atalar E. RF safety of wires in interventional MRI: using a safety index. Magn Reson Med 2002;47:187-193.

2. Langman DA, Goldberg IB, Judy J, Paul Finn J, Ennis DB. The dependence of radiofrequency induced pacemaker lead tip heating on the electrical conductivity of the medium at the lead tip. Magn Reson Med 2012;68:606-613.

3. Nordbeck P, Fidler F, Weiss I, et al. Spatial distribution of RF-induced E-fields and implant heating in MRI. Magn Reson Med 2008;60:312-319.

4. Nordbeck P, Weiss I, Ehses P, et al. Measuring RF-induced currents inside implants: impact of device configuration on MRI safety of cardiac pacemaker leads. Magn Reson Med 2009;61:570-578.

5. Mattei E, Calcagnini G, Censi F, Triventi M, Bartolini P. Role of the lead structure in MRI-induced heating: in vitro measurements on 30 commercial pacemaker/defibrillator leads. Magn Reson Med 2012;67: 925-935.
6. Langman DA, Goldberg IB, Finn JP, Ennis DB. Pacemaker lead tip heating in abandoned and pacemaker-attached leads at 1.5 Tesla MRI. J Magn Reson Imaging 2011;33:426-431.

7. Bonnemains L, Barbier T, Felblinger J. Metal wires should not be abandoned inside implantable cardioverter-defibrillators leads during heart transplantation! Transpl Int 2016;29:1136-1138.

8. Yeung CJ, Susil RC, Atalar E. RF heating due to conductive wires during MRI depends on the phase distribution of the transmit field. Magn Reson Med 2002;48:1096-1098.

9. Bottomley PA, Kumar A, Edelstein WA, Allen JM, Karmarkar PV. Designing passive MRI-safe implantable conducting leads with electrodes. Med Phys 2010;37:3828-3843.

10. E. Cabot, E. Zastrow, N. Kuster. Safety assessment of AIMDs under MRI exposure: tier3 vs. tier4 evaluation of local RF-induced heating. In Proceedings from the International Symposium on Electromagnetic Compatibility, Tokyo, Japan, 2014. pp 237-240.

11. ASTM F2182 - 11a. Standard test method for measurement of radio frequency induced heating on or near passive implants during magnetic resonance imaging. West Conshohocken, PA.

12. ISO/TS 10974:2012. Assessment of the safety of magnetic resonance imaging for patients with an active implantable medical device. Geneva, Switzerland.

13. Talcoth O, Rylander T. Electromagnetic modeling of pacemaker lead heating during MRI, 2011.

14. Park S-M, Kamondetdacha R, Nyenhuis JA. Calculation of MRIinduced heating of an implanted medical lead wire with an electric field transfer function. J Magn Reson Imaging 2007;26:1278-1285.

15. Feng S, Qiang R, Kainz W, Chen J. A technique to evaluate mriinduced electric fields at the ends of practical implanted lead. IEEE Trans Microw Theory Tech 2015;63:305-313.

16. Nyenhuis J, Jallal J, Min X, Sison S, Mouchawar G. In Proceedings from the Computing in Cardiology Conference, Sophia Antipolis, France, 2015. pp 765-768.

17. Nyenhuis J. MRI Bioeffects, Safety, and Patient Management, Chapter 21. Los Angeles, CA: Biomedical Research Publishing Group, 2014.

18. https://plastics.ulprospector.com/fr/generics/38/c/t/polyolefinepolyolefine-properties-processing. PROSPECTOR. Accessed May 25, 2017. 